\title{
Impaired atrial electromechanical function and atrial fibrillation promotion in alloxan-induced diabetic rabbits
}

\author{
Huaying Fu, Changle Liu, Jian Li, Changyu Zhou, \\ Lijun Cheng, Tong Liu, Guangping Li \\ Department of Cardiology, Tianjin Institute of Cardiology, \\ Second Hospital of Tianjin Medical University, Tianjin, People's Republic of China
}

\begin{abstract}
Background: Diabetes mellitus (DM) is an independent risk factor for atrial fibrillation $(A F)$. However, the underlying mechanisms are still not clearly elucidated. The aim of this study was to evaluate the atrial electromechanical function, atrial electrophysiological changes and $A F$ inducibility in alloxan-induced diabetic rabbits.
\end{abstract}

Methods: In 8 alloxan-induced diabetic rabbits and 8 controls, we evaluated atrial electromechanical function by tissue Doppler imaging. Isolated Langendorff-perfused rabbit hearts were prepared to measure atrial refractory effective period (AERP) and its dispersion (AERPD), interatrial conduction time (IACT) and vulnerability to AF. Atrial interstitial fibrosis was evaluated by Sirius-Red staining.

Results: Compared with controls, left atrial lateral wall Pa'-start interval (Pastart) and right atrial wall Pastart were increased in diabetic rabbits. AERPD was increased and IACT was prolonged in diabetic rabbits. Inducibility of $A F$ in diabetic group was significant higher than controls (6/8 vs. $1 / 8, p<0.05)$. Extensive interstitial fibrosis was observed in the DM group $(p<0.01)$. Correlation analysis showed that right atrial wall Pastart, $P a$ '-peak interval (Papeak) and total electromechanical activity (TEMA); left atrial lateral wall Papeak and TEMA, left atrial posterior wall TEMA, and IACT were correlated with atrial areas of fibrosis.

Conclusions: Atrial electromechanical function is impaired in diabetic rabbits, and is associated with atrial fibrosis and interatrial electrical conduction delay. (Cardiol J 2013; 20, 1: 59-67)

Key words: diabetes mellitus, atrial electromechanical function, atrial fibrillation

\section{Introduction}

Atrial fibrillation (AF) remains the most common clinically encountered arrhythmia associated with increased morbidity and mortality [1]. Diabetes mellitus (DM) has been reported as one of the strongest independent risk factors for $\mathrm{AF}$ and atrial flutter with an odds ratio of 1.4-2.2 [2-4] and its prevalence in AF patients is $10-25 \%$ [5]. On the other hand, DM is one of the most common chronic diseases with increasing prevalence of approximately 140 million people worldwide. This number is projected to increase to 300 million by the year of 2025 [6]. However, the pathophysiological pathways linking DM and $\mathrm{AF}$ remain obscure.

Address for correspondence: Tong Liu, MD, PhD; Guangping Li, MD, PhD, Department of Cardiology,

Tianjin Institute of Cardiology, Second Hospital of Tianjin Medical University, Tianjin 300211, People's Republic of China, tel: +86-22-88328368, fax: +86-22-28261158, e-mail: liutongdoc@yahoo.com.cn, e-mail: gp_limail@yahoo.com.cn

Received: 26.07.2012

Accepted: 08.10.2012 
The intra- and interatrial electromechanical delay (EMD) and the inhomogeneous propagation of sinus impulses are well-known electrophysiological characteristics of atrial remodeling that facilitate the development of $\mathrm{AF}$. This issue has been evaluated non-invasively by several alternative techniques such as $\mathrm{P}$-wave dispersion and tissue Doppler imaging (TDI) [7, 8]. However, data on the potential relationship between atrial EMD evaluated by TDI, atrial electrophysiological changes and $\mathrm{AF}$ promotion are sparse. The purpose of this study was to evaluate atrial EMD, left atrial (LA) mechanical functions, atrial electrophysiological changes and $\mathrm{AF}$ promotion, as well as to investigate whether atrial EMD and LA mechanical functions are related to atrial electrophysiological changes and $\mathrm{AF}$ promotion in alloxan-induced diabetic rabbits.

\section{Methods}

We obtained experimental animal use approval by the Experimental Animal Administration Committee of Tianjin Medical University and Tianjin Municipal Commission for Experimental Animal Control, which follow the guidelines established by the U.S. National Institutes of Health.

\section{Experimental animals}

Sixteen Japanese rabbits $(1.5-2.0 \mathrm{~kg}$ at the beginning of the study) (Beijing Medical Animals Research Institute, Beijing, China) were used in this study and divided into two groups: DM group $(\mathrm{n}=8)$ and control group $(\mathrm{n}=8)$. In the DM group, alloxan monohydrate (Sigma Aldrich Chemical, Saint Louis, MO, USA) was dissolved in sterile normal saline to achieve a concentration of $5 \%(\mathrm{~W} / \mathrm{V})$, and $120 \mathrm{mg} / \mathrm{kg}$ was immediately administered intravenously via the marginal ear vein over a period of 2 min with a 25 gauge butterfly catheter. The diabetic state was examined $48 \mathrm{~h}$ later by quantitative determination of blood glucose levels of $\geq 14 \mathrm{mmol} / \mathrm{L}$. Then, blood glucose concentration was monitored weekly with a glucometer Optium Xceed (Abbott Laboratories Medisense Products, Bedford, MA, USA). The animals in both groups were housed in cages in the Tianjin Institute of Cardiology under a $12 \mathrm{~h}$ light cycle, constant temperature and humidity, while they had free access to water and a standard laboratory pellet diet for 8 weeks.

\section{Echocardiographic examination}

After 8 weeks, echocardiographic images were obtained after inducing general anesthesia with $3 \%$ pelltobarbitalum natricum $(10 \mathrm{mg} / \mathrm{kg})$. Rabbits were placed on the table at the left lateral decubitus position. The echocardiograms were obtained using a GE Vingmed machine (Vivid 7/Vingmed General Electric, Milwaukee, WI) equipped with a $7.5 \mathrm{MHz}$ standard pediatric probe. Parasternal and apical views were obtained with the probe positioned at a cranial angle over a shaved area on the lower portion of the thoracic wall as previously reported [9, 10]. The exact position of the transducer was adjusted as necessary to acquire standard images. Standard parasternal short- and long-axis views as well as apical 2-, 3- and 4-chamber views acquired with at least 200 frames per second were digitized during 5 consecutive cardiac cycles in cine-loop format for analysis. Echocardiographic measurements by both $2 \mathrm{D}$ and $\mathrm{M}$-mode were obtained in the parasternal long axis view. These included interventricular septal thickness (IVST) and left ventricular posterior wall thickness (LVPWT), left ventricular end-diastolic dimension and end-systolic dimension, aortic root and left atrial anteroposterior diameter. Doppler imaging of the mitral valve was obtained from the apical 4-chamber view. The following measurements were obtained at the mitral inflow velocity image: peak $\mathrm{E}$ and peak $\mathrm{A}$ wave velocities, E/A ratio. Measurements of left ventricular ejection fraction (LVEF) according to international standards [11]. The average of the 3 measurements was used for subsequent analysis.

\section{Atrial mechanical functions}

Atrial mechanical functions comprised LA diameter measured from the parasternal long axis; all volume measurements were calculated from apical 4- and 2-chamber views using the biplane area-length method. Right atrial (RA) and LA maximal volume (immediately before the mitral valve opening), LA and RA minimal volume (at mitral valve closure), and LA and RA P-wave volume (RA and LA volumes at the beginning of the P-wave) were measured. LA and RA ejection fractions were calculated according to the formula (maximal volume - minimal volume)/ /maximal volume. LA and RA active emptying values were calculated as (volume at P-wave - minimal volume)/volume at $\mathrm{P}$-wave [12-14].

\section{Atrial electromechanical functions}

The TDI tracing was recorded over 5 cardiac cycles and was used for offline calculations. The different atrial walls were identified from the same apical views as follows: the RA wall, the interatrial septum, and the LA lateral wall from the apical 4-chamber view; the LA inferior wall and the LA anterior wall from the apical 2-chamber view; and 


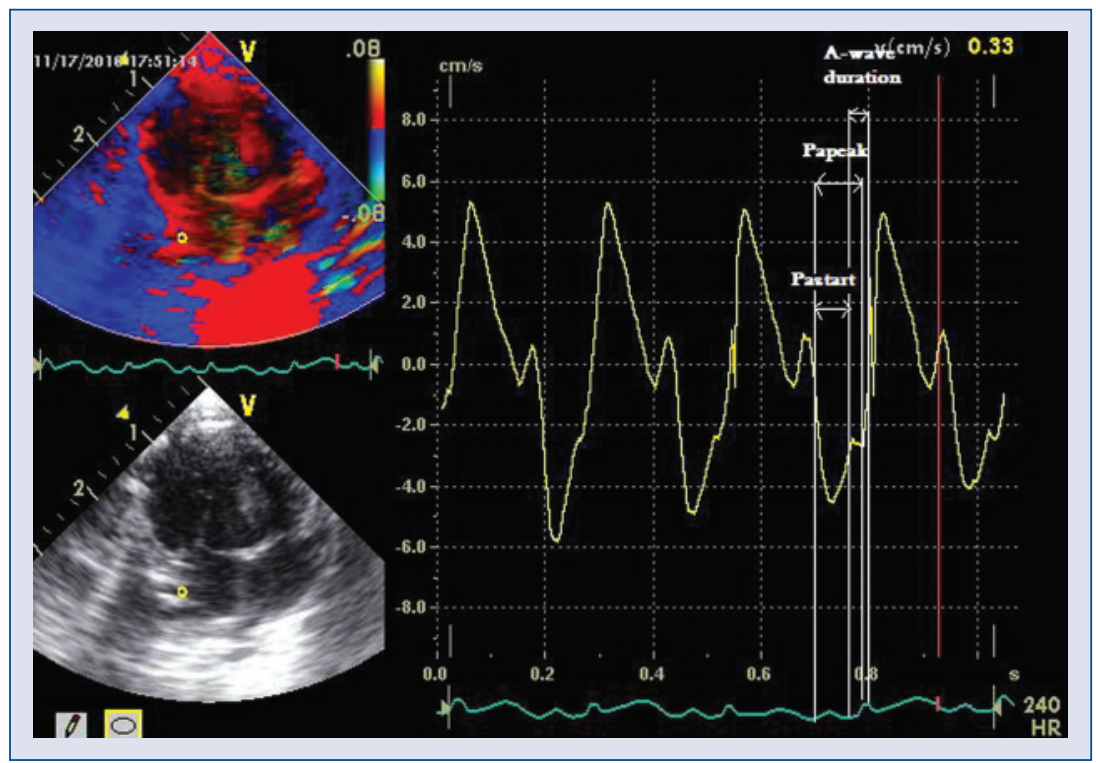

Figure 1. Representatives of measurements on atrial electromechanical function. Pastart was the time interval from the onset of $P$ wave on surface electrocardiogram (ECG) to the beginning of the $a^{\prime}$ wave on the TDI-curve profile; Papeak was the time interval from the onset of $P$ wave on surface ECG to the peak of the a' wave on the TDI-curve profile; The a'-wave duration was the time from the beginning to the end of the $a^{\prime}$ wave on the TDI-curve profile. The total electromechanical activity was the time between the beginnings of the P-wave on the monitor ECG to the end of the $a^{\prime}$ wave on the TDI-curve profile.

the LA posterior wall (post) from the apical 3-chamber view. Each atrial wall was studied at mid levels, placing a $1 \mathrm{~mm}$ sample volume at the mid portion of each atrial wall. The regional electromechanical function at each atrial wall was studied by the following time intervals. The Pa'-start interval (Pastart) was defined as the time between the beginning of the $\mathrm{P}$-wave on the monitor's electrocardiogram (ECG) (lead II) to the start of the a' wave on the TDI-curve profile. The Pa'-peak interval (Papeak) was the time between the beginning of the P-wave on the monitor's ECG to the peak of the a' wave on the TDI-curve profile. The a'-wave duration (a-dur) was the time from the beginning to the end of the a' wave on the TDI-curve profile. The total electromechanical activity (TEMA) was the time between the beginnings of the $\mathrm{P}$-wave on the monitor ECG to the end of the a' wave on the TDI-curve profile. Expressions of atrial electromechanical function shown in Figure 1.

\section{Hemodynamic studies}

At 8 weeks, the rabbits were intravenously heparinized (1000 IU) and anesthetized with 3\% pelltobarbitalum natricum $(30 \mathrm{mg} / \mathrm{kg})$. The ECG tracing was obtained with bipolar cutaneous electrodes placed on all 4 limbs and the signal was amplified and filtered. The right carotid artery was iso- lated surgically with a median incision in the neck. Cannulae was inserted into the right carotid artery to monitor aortic systolic and diastolic blood pressure (SBP, DBP). SBP and DBP (3 times, $20 \mathrm{~s}$ interval) were recorded after a stabilization period of 5-10 min. Then the cannulae was moved slowly through the aortic valve to left ventricle to measure left ventricle end-diastolic pressure (LVEDP). The ECG and BP were continuously monitored on custom-made computer software program (Electrophysiological Recording System, TOP-2001, HTONG Company, Shanghai, China).

\section{Electrophysiological studies}

Following the hemodynamic studies, the rabbit hearts were quickly removed and placed in cold perfusion fluid $\left(4^{\circ} \mathrm{C}\right)$. The aorta was cannulated and connected to a Langendorff perfusion system filled with warmed $\left(37 \pm 0.5^{\circ} \mathrm{C}\right)$ Tyrode' s solution. The perfusion pressure was maintained at $65-75 \mathrm{~mm} \mathrm{Hg}$, resulting in an initial coronary flow of 50 to $60 \mathrm{~mL} / \mathrm{min}$. The Tyrode's solution consisted of the following (mmol/L): $\mathrm{NaCl} 130, \mathrm{KCl} 5.6, \mathrm{NaHCO}_{3}$ 24.2, $\mathrm{CaCl}_{2}$ 2.2, $\mathrm{MgCl}_{2}$ 0.6, $\mathrm{NaH}_{2} \mathrm{PO}_{4} 1.2$, and glucose 12 . The solution reservoir was saturated with $95 \% \mathrm{O}_{2}$ and $5 \% \mathrm{CO}_{2}$ and had a $\mathrm{pH}$ of 7.4.

Four sets of silver bipolar electrodes were placed on the epicardial surface of high right 
atrium (HRA), high left atrium (HLA), left atrial appendage and right ventricular apex. The atrial effective refractory period (AERP) was evaluated using programmed extra-stimuli and defined as the longest S1-S2 interval that failed to capture. The S2 extra-stimulus was delivered after a drive-train of $8 \mathrm{~S} 1$ stimuli of $250 \mathrm{~ms}$. The S1-S2 interval was decreasing in intervals of $2 \mathrm{~ms}$ until atrial refractoriness was reached (pulse duration: $2 \mathrm{~ms}$, output: 2 -fold diastolic pacing threshold). AERP dispersion (AERPD) was defined as the difference between the longest AERP and the shortest AERP at 4 different sites. Wenckebach cycle length of atrial-ventricular conduction was measured by RA incremental pacing. The interatrial conduction time (IACT) was measured during HRA (at a pacing cycle length of $250 \mathrm{~ms}$ ) pacing. IACT was defined as the duration from the HRA pacing stimulus to the beginning of HLA stimulus. AF vulnerability was tested by the burst pacing (cycle length of $50 \mathrm{~ms}$ ) for $1 \mathrm{~s}, 5$ times at $30 \mathrm{~s}$ interval at an amplitude of $5 \mathrm{~V}$. We evaluated the appearance of $\mathrm{AF}$, defined as rapid, irregular atrial response longer than $1000 \mathrm{~ms}$. All epicardial ECG were amplified with a custom-made amplifier and recorded with a custom-made computer software program (Electrophysiological Recording System, TOP-2001, HTONG Company, Shanghai, China).

\section{Histological studies}

Isolated atrial samples from the LA free wall was placed in $4 \%$ paraformaldehyde, embedded in paraffin, and cut into $4 \mu \mathrm{m}$ cross-sections. Sirius-Red (Sigma Aldrich) staining was used to evaluate interstitial fibrosis. We randomly selected $40 \mathrm{sec}-$ tions from 8 rabbits per group and evaluated them at 200 -fold magnification. The vascular and perivascular fibrosis were excluded from the selected sections. The red pixel content of digitized images was detected as interstitial fibrotic area and measured relative to total tissue area with a digital imaging analyzer of Image Pro Plus 4.5 Scion image software (Scion corporation, Frederick, MD), these analyses were performed in a blind manner. The result was obtained from 2 independent measurements.

\section{Statistical analyses}

Data are presented as mean \pm standard deviation (SD). Statistical analysis was done using the Student's t-test (unpaired). The incidence of AF was analyzed by Fisher's exact test. Statistical differences were considered significant at a $\mathrm{p}$ value $<0.05$. For the exploration of relationships between TDI parameters, electrophysiological parameters and atrial interstitial fibrosis, we calculated Pearson's correlation coefficients.

\section{Results}

\section{Echocardiographic characteristics}

Left atrial mechanical functions. LA volume parameters are presented in Table 1 . Both groups were similar with respect to maximum LA volume, LA passive emptying volume, LA passive emptying fraction and LA total emptying volume. However, LA diameter, IVST, LVPWT were significantly increased in DM group compared with controls.

Atrial electromechanical function. The atrial electromechanical function parameters measured from different sites by TDI are shown in Table 2 . RA Pastart, LA lateral wall Pastart, LA lateral wall Papeak and LA posterior wall TEMA were greater in the DM group compared with controls $(\mathrm{p}<0.05)$. Pastarts and Papeaks in other atrial walls tended to increase in DM group, but they did not reach statistical significance.

\section{Hemodynamic parameters}

As shown in Table 3, the DBP and LVEDP were slightly increased in DM group. However, no significant differences were observed between the 2 groups.

\section{Electrophysiological studies}

As shown in Table 3, in both the RA and the LA, the AERPs in DM group were slightly prolonged. However, no significant differences were observed between the 2 groups. AERPD was significantly increased in DM group ( $\mathrm{p}<0.001)$. IACT in diabetic rabbits was significantly prolonged compared with healthy controls. Figure 2A shows representative $\mathrm{AF}$ induced by the burst pacing, which were observed in the DM group; Figure $2 \mathrm{~B}$ shows the inducibility of $\mathrm{AF}$ in both groups. AF was induced in 6/8 (75\%) of animals in DM group, whereas $1 / 8(12.5 \%)$ in controls $(\mathrm{p}<0.05)$.

\section{Atrial interstitial fibrosis}

Figure $3 \mathrm{~A}$ and $\mathrm{B}$ illustrates the representative LA histology in both groups and the quantitative ratio of the area of fibrosis to the area of the reference tissue is summarized in Figure 3C. Compared with the control group, extensive and heterogeneous interstitial fibrosis was observed in the DM group $(2.09 \pm 0.7 \%$ vs. $6.67 \pm 2.3 \%, \mathrm{p}<0.01)$. 
Table 1. Basic characteristics and atrial mechanical functions.

\begin{tabular}{|c|c|c|c|}
\hline & DM group (n = 8) & Control group $(n=8)$ & $\mathbf{P}$ \\
\hline Gender (male/female) & $4 / 4$ & $3 / 5$ & 1.00 \\
\hline Weight [kg] & $2.29 \pm 0.47$ & $2.19 \pm 0.17$ & 0.55 \\
\hline Glucose level at $8 \mathrm{~W}[\mathrm{mmol} / \mathrm{L}]$ & $16.35 \pm 3.44$ & $5.81 \pm 0.81$ & $<0.001$ \\
\hline LAD [mm] & $8.28 \pm 0.58$ & $6.75 \pm 0.59$ & $<0.001$ \\
\hline IVST [mm] & $2.95 \pm 0.54$ & $1.91 \pm 0.20$ & $<0.001$ \\
\hline LVPWT [mm] & $2.98 \pm 0.39$ & $1.92 \pm 0.17$ & $<0.001$ \\
\hline LVEDD [mm] & $11.86 \pm 0.75$ & $12.27 \pm 1.33$ & 0.44 \\
\hline LVESD [mm] & $7.55 \pm 1.21$ & $7.71 \pm 1.20$ & 0.16 \\
\hline LV ejection fraction [\%] & $71.33 \pm 5.52$ & $73.00 \pm 5.04$ & 0.53 \\
\hline LA maximal volume $\left[\mathrm{mL} / \mathrm{m}^{2}\right]$ & $0.48 \pm 0.09$ & $0.45 \pm 0.17$ & 0.64 \\
\hline LA minimal volume $\left[\mathrm{mL} / \mathrm{m}^{2}\right]$ & $0.17 \pm 0.08$ & $0.14 \pm 0.06$ & 0.40 \\
\hline LA P-wave volume $\left[\mathrm{mL} / \mathrm{m}^{2}\right]$ & $0.25 \pm 0.10$ & $0.21 \pm 0.07$ & 0.34 \\
\hline LA ejection fraction [\%] & $59.44 \pm 6.04$ & $59.12 \pm 8.62$ & 0.93 \\
\hline LA active emptying [\%] & $34.66 \pm 6.63$ & $33.87 \pm 7.60$ & 0.82 \\
\hline RA maximal volume $\left[\mathrm{mL} / \mathrm{m}^{2}\right]$ & $0.29 \pm 0.13$ & $0.29 \pm 0,12$ & 0.93 \\
\hline $\mathrm{RA}$ minimal volume $\left[\mathrm{mL} / \mathrm{m}^{2}\right]$ & $0.16 \pm 0.06$ & $0.12 \pm 0.05$ & 0.20 \\
\hline RA P-wave volume $\left[\mathrm{mL} / \mathrm{m}^{2}\right]$ & $0.22 \pm 0.06$ & $0.16 \pm 0.09$ & 0.16 \\
\hline RA ejection fraction [\%] & $47.33 \pm 9.72$ & $52.5 \pm 3.63$ & 0.37 \\
\hline RA active emptying [\%] & $23.55 \pm 5.70$ & $30.62 \pm 8.01$ & 0.05 \\
\hline E velocity $[\mathrm{cm} / \mathrm{s}]$ & $46.77 \pm 15.25$ & $64.75 \pm 3.38$ & 0.07 \\
\hline A velocity $[\mathrm{cm} / \mathrm{s}]$ & $51.77 \pm 12.71$ & $54.95 \pm 18.92$ & 0.68 \\
\hline$E / A$ & $1.29 \pm 0.64$ & $0.99 \pm 0.31$ & 0.43 \\
\hline $\mathrm{E} / \mathrm{e}^{\prime}$ & $9.03 \pm 2.77$ & $10.29 \pm 4.33$ & 0.26 \\
\hline
\end{tabular}

Values are mean \pm SD; DM — diabetes; LAD — left atrial diameter; IVST — interventricular septal thickness; LVPWT — left ventricular posterior wall thickness; LVEDD - left ventricular end-diastolic dimension; LVESD — left ventricular end-systolic dimension; LV — left ventricular; LA — left atrial; RA — right atrial; E velocity - early diastolic velocity of mitral inflow; A velocity — late diastolic velocity of mitral inflow; $\mathrm{e}^{\prime}$ — early diastolic velocity of septal mitral annulus

Table 2. Atrial electromechanical function assessed by Doppler tissue echocardiography.

\begin{tabular}{|c|c|c|c|}
\hline & DM group $(n=8)$ & Control group $(n=8)$ & $\mathbf{P}$ \\
\hline \multicolumn{4}{|c|}{ Right atrial wall } \\
\hline Pastart [ms] & $60.44 \pm 20.49$ & $40.50 \pm 12.87$ & 0.032 \\
\hline Papeak [ms] & $82.44 \pm 24.53$ & $62.12 \pm 18.26$ & 0.075 \\
\hline TEMA $[\mathrm{ms}]$ & $102.44 \pm 20.59$ & $91.75 \pm 19.19$ & 0.287 \\
\hline \multicolumn{4}{|c|}{ Left atrial lateral wall } \\
\hline Pastart [ms] & $60.55 \pm 14.36$ & $39.75 \pm 18.32$ & 0.019 \\
\hline Papeak [ms] & $83.00 \pm 11.02$ & $59.75 \pm 20.09$ & 0.009 \\
\hline TEMA [ms] & $106.33 \pm 15.43$ & $92.62 \pm 20.80$ & 0.141 \\
\hline \multicolumn{4}{|c|}{ The interatrial septum } \\
\hline Pastart [ms] & $54.66 \pm 21.02$ & $46.87 \pm 21.78$ & 0.465 \\
\hline Papeak [ms] & $75.77 \pm 21.94$ & $64.12 \pm 23.24$ & 0.305 \\
\hline TEMA $[\mathrm{ms}]$ & $98.66 \pm 22.89$ & $97.87 \pm 19.98$ & 0.941 \\
\hline \multicolumn{4}{|c|}{ Left atrial anterior wall } \\
\hline Pastart [ms] & $69.55 \pm 16.80$ & $67.50 \pm 16.30$ & 0.802 \\
\hline Papeak [ms] & $85.66 \pm 15.26$ & $87.25 \pm 13.39$ & 0.824 \\
\hline TEMA [ms] & $131.56 \pm 67.97$ & $108.50 \pm 15.21$ & 0.364 \\
\hline \multicolumn{4}{|c|}{ Left atrial inferior wall } \\
\hline Pastart [ms] & $57.11 \pm 24.96$ & $53.87 \pm 19.21$ & 0.771 \\
\hline Papeak [ms] & $78.55 \pm 23.11$ & $70.25 \pm 20.88$ & 0.451 \\
\hline TEMA [ms] & $109.11 \pm 23.97$ & $96.62 \pm 15.86$ & 0.231 \\
\hline \multicolumn{4}{|c|}{ Left atrial posterior wall } \\
\hline Pastart [ms] & $62.11 \pm 25.42$ & $46.87 \pm 12.47$ & 0.146 \\
\hline Papeak [ms] & $75.66 \pm 30.95$ & $60.87 \pm 9.99$ & 0.217 \\
\hline TEMA [ms] & $104.67 \pm 22.38$ & $83.12 \pm 6.10$ & 0.019 \\
\hline
\end{tabular}

Values are presented as mean \pm SD; DM - diabetes; Pastart - the time between the beginning of the P-wave on the monitor's ECG to the start of the $a^{\prime}$ wave on the DTE-curve profile; Papeak — the time between the beginning of the P-wave on the monitor's ECG to the peak of the a' wave on the DTE-curve profile; TEMA - total electromechanical activity 
Table 3. Hemodynamic and electrophysiological studies.

\begin{tabular}{lccc}
\hline & DM $(\mathbf{n}=\mathbf{8})$ & Control $(\mathbf{n}=\mathbf{8})$ & P \\
\hline SBP $[\mathrm{mm} \mathrm{Hg}]$ & $119.5 \pm 6.83$ & $124.7 \pm 10.23$ & 0.3 \\
DBP [mm Hg] & $102.16 \pm 4.79$ & $97.12 \pm 7.60$ & 0.18 \\
LVEDP [mm Hg] & $-5.80 \pm 8.10$ & $-8.57 \pm 3.59$ & 0.436 \\
HR [bpm] & $152.33 \pm 33.99$ & $169.89 \pm 16.31$ & 0.182 \\
IACT [ms] & $37.66 \pm 8.88$ & $27.70 \pm 2.12$ & 0.005 \\
AVWCL [ms] & $186.00 \pm 44.81$ & $165.44 \pm 9.28$ & 0.197 \\
HRAERP [m]s & $98.00 \pm 28.96$ & $81.55 \pm 5.83$ & 0.115 \\
HLAERP [ms] & $99.12 \pm 34.39$ & $79.11 \pm 7.88$ & 0.109 \\
LLAERP [ms] & $104.12 \pm 38.67$ & $82.44 \pm 12.12$ & 0.130 \\
AERPD [ms] & $28.37 \pm 7.52$ & $11.62 \pm 5.60$ & $<0.001$ \\
\hline
\end{tabular}

Values are mean \pm SD, DM - diabetes; SBP — systolic blood pressure, DBP — diastolic blood pressure, LVEDP — left ventricular end diastolic pressure, $\mathrm{HR}$ - heart rate; IACT — interatrial conduction time; AVWCL — Wenckebach cycle length of A-V conduction; HRAERP - high right atrial effective refractory period; HLAERP — high left atrial effective refractory period; LLAERP — low left atrial effective refractory period; AERPD — atrial effective refractory periods dispersion

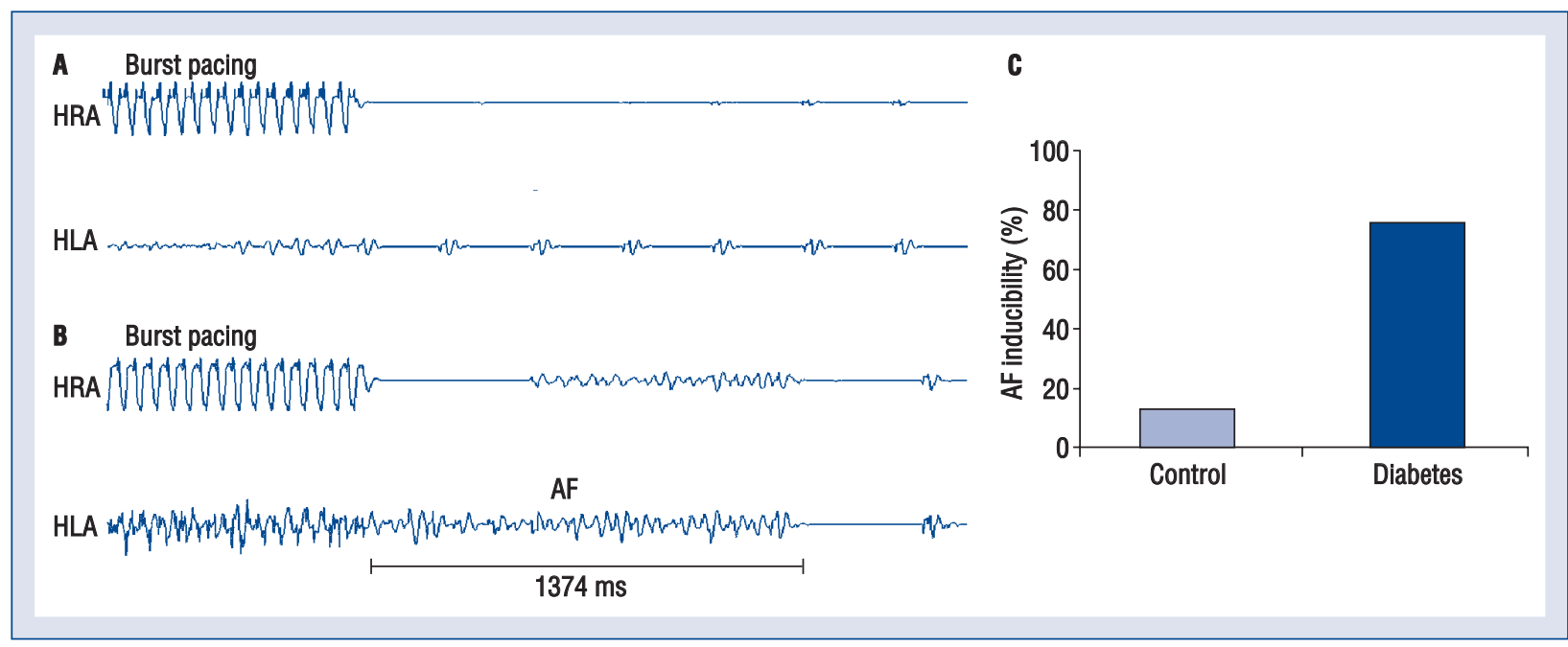

Figure 2. Atrial fibrillation (AF) inducibility in both groups; A. Representative AF episode induced during Burst pacing in diabetes mellitus group; B. Inducibility of AF in both groups; C. Comparison of the inducibility of AF between the diabetes group and controls; $n=8$ in each group; $p<0.01$; HRA - high right atrium; HLA - high left atrium.
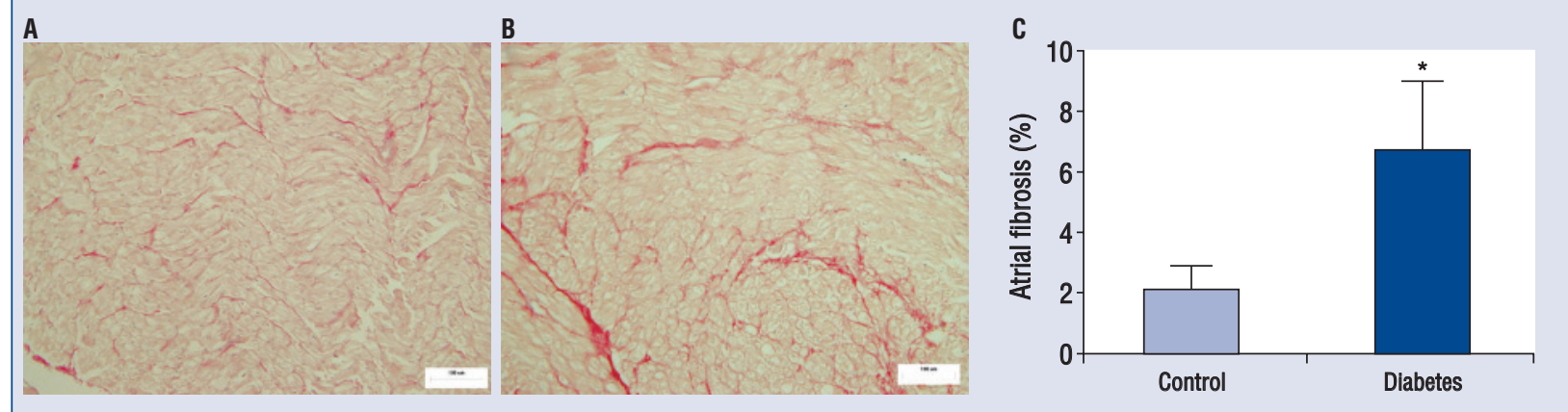

Figure 3. Histological examinations on left atrial fibrosis in both groups; A, B. Representative Sirius-Red staining of the two groups; C. Comparsion of the mean percentage of left atrial interstitial fibrosis in each group. Data are presented as mean $\pm S D ; n=8$ in each group; ${ }^{*} p<0.01$. 

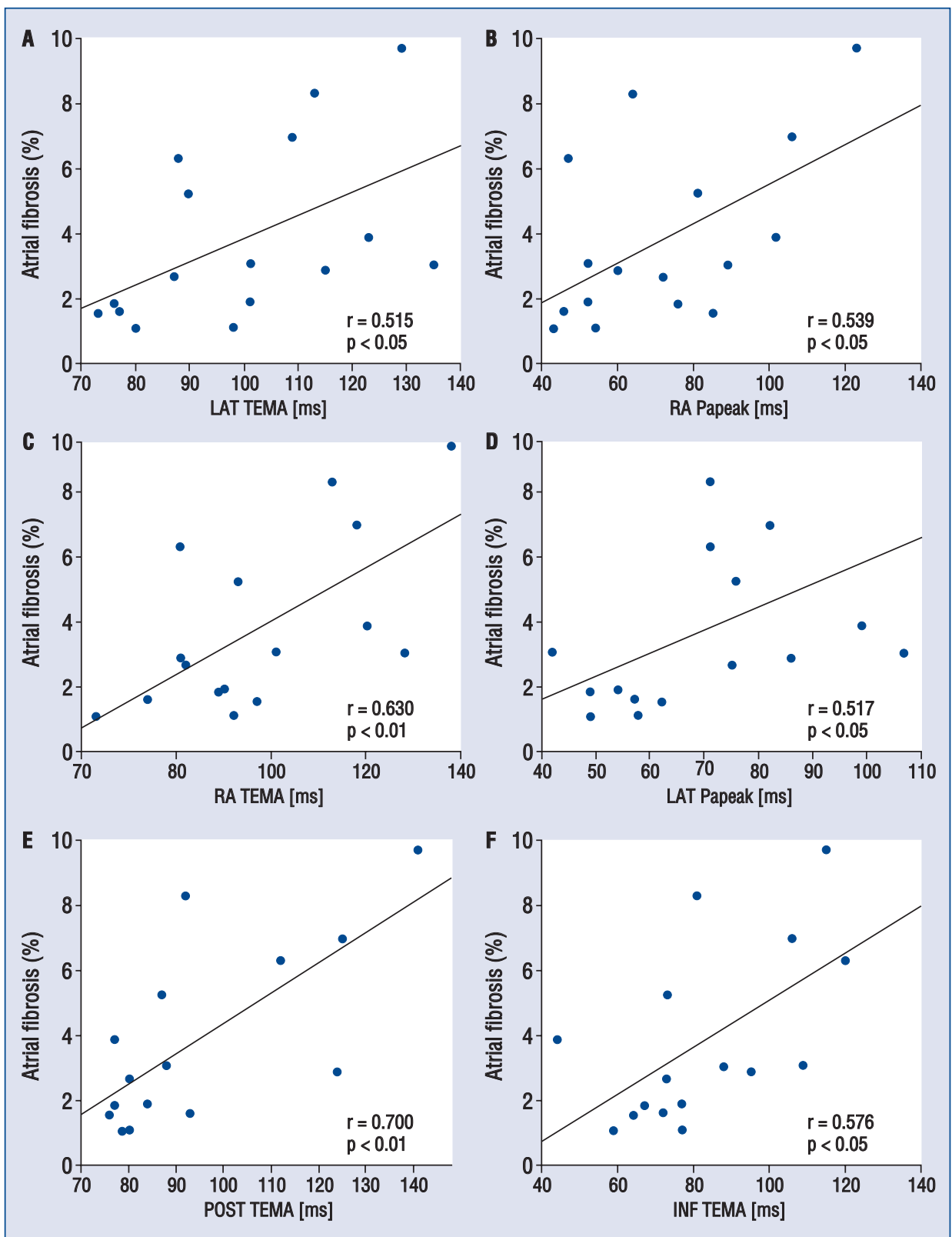

Figure 4. Relationship between atrial interstitial fibrosis and atrial electromechanical function; TEMA — total electromechanical activity; LAT — left atrial lateral wall; Papeak — Pa'-peak interval; RA — right atrial wall; POST — left atrial posterior wall; INF — left atrial inferior wall

\section{Relationship between atrial interstitial} fibrosis and atrial electromechanical function

Correlation analysis showed that RA wall Pastart, Papeak and TEMA, LA lateral wall Papeak and TEMA, LA posterior wall TEMA, LA inferior wall TEMA, blood glucose levels, IACT were correlated with atrial areas of fibrosis $(\mathrm{r}=0.507, \mathrm{p}<0.05$; $\mathrm{r}=0.539, \mathrm{p}<0.05 ; \mathrm{r}=0.630, \mathrm{p}<0.01 ; \mathrm{r}=0.517$, $\mathrm{p}<0.05 ; \mathrm{r}=0.515, \mathrm{p}<0.05 ; \mathrm{r}=0.700, \mathrm{p}<0.01$; $\mathrm{r}=0.576, \mathrm{p}<0.05 ; \mathrm{r}=0.700, \mathrm{p}<0.01 ; \mathrm{r}=0.606$, $\mathrm{p}<0.05$, respectively) (Fig. 4). Correlation analy- sis also showed that inter-atrial septum Pastart, LA posterior wall Pastart and LA posterior wall Papeak were correlated with IACT $(\mathrm{r}=0.634,0.562,0.629$, respectively, $\mathrm{p}<0.05)$.

\section{Discussion}

\section{Major findings}

The major findings of the present study were as follows: (1) Atrial electromechanical dysfunction was present in the alloxan-induced diabetic rabbits. 
(2) Alloxan-induced diabetic rabbit atria showed structural remodeling characterized by diffuse interstitial fibrosis. (3) Atrial electrophysiological changes in diabetic hearts included prolonged interatrial electrical conduction and increased AERP dispersion. (4) Impaired atrial electromechanical function, LA interstitial fibrosis and interatrial electrical conduction delay were correlated, and may constitute a substrate for the development of $\mathrm{AF}$ in diabetic atria.

\section{Atrial electromechanical dysfunction in diabetes mellitus}

Previous studies have showed that intra- and interatrial electromechanical coupling and atrial EMD, measured by TDI, were increased in patients with paroxysmal AF and type $1 \mathrm{DM}$ compared with healthy controls $[8,15]$. These data suggest that increased interatrial EMD might be related to an increased risk of $\mathrm{AF}[8,15]$. Acar et al. [15] evaluated atrial electromechanical coupling obtained by TDI in patients with type $1 \mathrm{DM}$, indicating that intra- and inter-atrial EMD are prolonged, whereas diastolic functions of both ventricles and LA mechanical functions are impaired in patients with type $1 \mathrm{DM}$. However, intra- and inter-atrial conduction times were not investigated by electrophysiological techniques. Our present study also showed that atrial electromechanical function were impaired in diabetic rabbits. In particular, the parameters regarding atrial electromechanical function were closely related to the extent of atrial interstitial fibrosis and interatrial electrical conduction time. To the best of our knowledge, our study was the first to evaluate the potential relationship between the echocardiographic LA electromechanical function, atrial interstitial fibrosis and interatrial electrical conduction delay in diabetic rabbit hearts.

\section{Atrial mechanical functions in diabetes mellitus}

A recent study indicated LA mechanical function measured by LA total emptying fraction is a predictor for the development of post-operative $\mathrm{AF}$ following cardiac surgery [16]. Additionally, the atrial emptying pattern is strongly affected by LV diastolic properties $[17,18]$. It has been demonstrated that in diabetic patients, decreased LA passive emptying volume is related to increased LVEDP and increased LA active emptying volume is associated with compensatory mechanisms in LA contraction [15]. However, in present study, diastolic and systolic dysfunction of LV and systolic dysfunction of LA were not observed. Moreover, hemodynamic parameters, such as the DBP and LVEDP were slightly increased in DM group. Therefore, we speculate that diabetic atrial mechanical dysfunction at 8 week was too slight to be detected by echocardiography.

\section{Atrial substrate and remodeling in diabetes mellitus}

Atrial remodeling involves changes in the structure, function and geometry of the atria, modification of atrial electrical and contractile properties, and changes in the amount and the composition of the extracellular matrix [19, 20]. Together, these alterations create an arrhythmogenic substrate essential for the onset and persistence of AF. Structural remodeling results in electrical tissue non-homogeneity, slowed conduction and electrical uncoupling, facilitating AF continuation without inducing changes in atrial action potential properties.

The potential mechanisms for diabetes-induced atrial remodeling were not fully understood. It was supposed that inflammation, oxidative stress activation, autonomic imbalance were observed in diabetic pathophysiological state, which may promote the development of AF. Also angiotensin 1 receptor activity was upregulated in diabetic hearts [21], and this receptor was coupled with transforming growth factor-beta1 and lead to atrial fibrosis [22]. Kato et al. [23] showed that inter-atrial conduction delay and increased fibrotic deposition in atrium play a major role in producing atrial arrhythmogenicity in genetic type $2 \mathrm{DM}$ rats, which indicated that atrial structural remodeling characterized by extensive interstitial fibrosis may be one of the major mechanisms of $\mathrm{AF}$ development in diabetic heart. Another study [24] from this group demonstrated that activation of advanced glycation end products and its receptor was associated with diffuse atrial interstitial fibrosis in streptozotocin-induced diabetic rats. The present study showed that impaired LA electromechanical function along with extensive atrial fibrosis and increased inter-atrial conduction delay were related to increased inducibility of AF. Also, our results also indicated that AERP dispersion was increased in DM rabbits, suggesting that heterogeneity of repolarization is an electrophysiological change in this setting. However, the molecular mechanisms for DM-induced atrial electromechanical dysfunction and atrial remodeling should be investigated in future studies. Further studies on atrial electromechanical function may be of special value to assess the impact of novel thearpeutic interventions anti-remodeling effects such as glitazones [25] on prevention and treatment of $\mathrm{AF}$. 


\section{Limitations of the study}

There are several limitations in the present study. First, tissue Doppler measurements depend on skills of the person performing the examinations and need suitable acoustic window to carry out registrations of acceptable quality. We have to take into account the intrinsic limitations of the technique, such as dependency on the angle of incidence, the variable position of the sample volume during the cardiac cycle, and the complex movement of the analyzed segment. Heart rate and small heart of the rabbit may affect the imaging quality of our study. Second, we do not have data on the successive electrophysiological and structural changes over time as well as on the temporal relationship between the two types of remodeling. Another limitation is that we did not assess the possible paradoxical response of AERP to increased heart rates which is another characteristic of electrical remodeling. Finally, the molecular mechanisms for DM-induced electrical and structural remodeling should be further investigated in future studies.

\section{Acknowledgements}

This work was supported by grant (No. 30900618 to T.L.) from the Program of Natural Science Foundation of China. The authors thank Dr. Panagiotis Korantzopoulos (Department of Cardiology, University of Ioannina School of Medicine, Ioannina, Greece) for helpful comments and suggestions.

\section{Conflicts of interest: none declared}

\section{References}

1. Kannel WB, Benjamin EJ. Current perceptions of the epidemiology of atrial fibrillation. Cardiol Clin, 2009; 27: 13-24.

2. Huxley RR, Filion KB, Konety S, Alonso A. Meta-analysis of cohort and case-control studies of type 2 diabetes mellitus and risk of atrial fibrillation. Am J Cardiol, 2011; 108: 56-62.

3. Movahed MR, Hashemzadeh M, Jamal MM. Diabetes mellitus is a strong, independent risk for atrial fibrillation and flutter in addition to other cardiovascular disease. Int J Cardiol, 2005; 105: 315-321.

4. Lip GYH, Varughese GI. Diabetes mellitus and atrial fibrillation: Perspectives on epidemiological and pathophysiological links. Int J Cardiol, 2005; 105: 319-321.

5. KrahnAD, Manfreda J, Tate RB, Mathewson FA, Cuddy TE. The natural history of atrial fibrillation: Incidence, risk factors, and prognosis in the Manitoba Follow-Up Study. Am J Med, 1995; 98: $476-484$.

6. King H, Aubert RE, Herman WH. Global burden of diabetes, 1995-2025: prevalence, numerical estimates, and projections. Diabetes Care, 1998; 21: 1414-1431.

7. Dilaveris PE, Gialafos EJ, Sideris SK et al. Simple electrocardiographic markers for the prediction of paroxysmal idiopathic atrial fibrillation. Am Heart J, 1998; 135: 733-738.
8. Cui QQ, Zhang W, Wang $\mathrm{H}$ et al. Assessment of atrial electromechanical coupling and influential factors in nonrheumatic paroxysmal atrial fibrillation. Clin Cardiol, 2008; 31: 74-78.

9. Nagueh SF, Kopelen HA, Lim DS et al. Tissue Doppler imaging consistently detects myocardial contraction and relaxation abnormalities, irrespective of cardiac hypertrophy, in a transgenic rabbit model of human hypertrophic cardiomyopathy. Circulation, 2000; 102: 1346-1350.

10. Fontes-Sousa APN, Bras-Silva C, Moura C, Areias JC, Leite-Moreira AF. M-mode and Doppler echocardiographic reference values for male New Zealand white rabbits. Am J Vet Res, 2006; 67: 1725-1729.

11. Schiller NB, Shah PM, Crawford M et al. Recommendations for quantitation of the left ventricle by two dimensional echocardiography. American Society of Echocardiography Committee on standards, subcommittee on quantitation of two-dimensional echocardiograms. J Am Soc Echocardiogr, 1989; 2: 358-367.

12. Paraskevaidis IA, Dodouras T, Adamopoulos S, Kremastinos DT. Left atrial functional reserve in patients with nonischemic dilated cardiomyopathy: An echocardiographic dobutamine study. Chest, 2002; 122: 1340-1347.

13. Triposkiadis F, Moyssakis I, Hadjinikolaou L et al. Left atrial systolic function is depressed in idiopathic and preserved in ischemic dilated cardiomyopathy. Eur J Clin Invest, 1999; 29: 905-912.

14. Kircher B, Abbott JA, Pau S et al. Left atrial volume determination by biplane two-dimensional echocardiography: Validation by cine computed tomography. Am Heart J, 1991; 121: 864-871.

15. Acar G, Akcay A, Sokmen A et al. Assessment of atrial electromechanical delay, diastolic functions, and left atrial mechanical functions in patients with type 1 diabetes mellitus. J Am Soc Echocardiogr, 2009; 22: 732-738.

16. Haffajee JA, Lee Y, Alsheikh-Ali AA, Kuvin JT, Pandian NG, Patel AR. Pre-operative left atrial mechanical function predicts risk of atrial fibrillation following cardiac surgery. J Am Coll Cardiol Imaging, 2011; 4: 833-840.

17. Aktoz M, Yilmaztepe M, Tatli E, Turan FN, Umit EG, Altun A. Assessment of ventricular and left atrial mechanical functions, atrial electromechanical delay and $\mathrm{P}$ wave dispersion in patients with scleroderma. Cardiol J, 2011; 18: 261-269.

18. Henein M, Zhao Y, Henein MY, Lindqvist P. Disturbed left atrial mechanical function in paroxysmal atrial fibrillation: A speckle tracking study. Int J Cardiol, 2012; 155: 437-441.

19. Aldhoon B, Melenovsky V, Peichl P, Kautzner J. New insights into mechanisms of atrial fibrillation. Physiol Res, 2010; 59: 1-12.

20. Burstein B, Nattel S. Atrial fibrosis: Mechanisms and clinical relevance in atrial fibrillation. J Am Coll Cardiol, 2008; 51: 802-809.

21. Hotta H, Miura T, Miki T et al. Angiotensin II type 1 receptormediated upregulation of calcineurin activity underlies impairment of cardioprotective signaling in diabetic hearts. Circ Res, 2010; 106: 129-132

22. Rosenkranz S. TGF-beta1 and angiotensin networking in cardiac remodeling. Cardiovasc Res, 2004; 63: 423-432.

23. Kato T, Yamashita T, Sekiguchi A et al. What are arrhythmogenic substrates in diabetic rat atria? J Cardiovasc Electrophysiol, 2006; 17: 890-894.

24. Kato T, Yamashita T, Sekiguchi A et al. AGEs-RAGE system mediates atrial structural remodeling in the diabetic rat. J Cardiovasc Electrophysiol, 2008; 19: 415-420.

25. Liu T, Korantzopoulos P, Li G, Li J. The potential role of thiazolidinediones in atrial fibrillation. Int J Cardiol, 2008; 128: 129-130. 